\title{
The Utility of Diagnostic Imaging in Fish Bone Impaction
}

\author{
Shanesh Kumar*, Casey Yu, Jason Toppi, Matthew Ng, Fiona Hill, Natalie Sist \\ Department of Otolaryngology, Box Hill Hospital, Victoria, Australia \\ Email: *shaneshkumar@outlook.com
}

How to cite this paper: Kumar, S., Yu, C., Toppi, J., Ng, M., Hill, F. and Sist, N. (2018) The Utility of Diagnostic Imaging in Fish Bone Impaction. Open Journal of Radiology, 8, 45-52. https://doi.org/10.4236/ojrad.2018.81006

Received: January 2, 2018

Accepted: March 11, 2018

Published: March 14, 2018

Copyright ( 2018 by authors and Scientific Research Publishing Inc. This work is licensed under the Creative Commons Attribution International License (CC BY 4.0).

http://creativecommons.org/licenses/by/4.0/

(c) (i) Open Access

\begin{abstract}
Objectives: The purpose of this pilot study was to obtain an Australian perspective on evaluating the utility of plain film radiography and computed tomography (CT) to rule out fish bone impaction in the upper aerodigestive tract in the emergency department (ED) setting. Methods: A retrospective multicentre cohort study was conducted. A total of 73 patients met the inclusion criteria. A subgroup of patients underwent CT. We studied the sensitivity and specificity of $\mathrm{x}$-ray and $\mathrm{CT}$ along with other demographic variables to determine the likelihood of true fish bone impaction. Results: Out of the 73 patients, 28 patients had true bone impaction. The sensitivity for $\mathrm{x}$-ray was $42.9 \%$ and specificity was $73.3 \%$. The sensitivity of CT was $87.5 \%$ and specificity was $71.4 \%$. We found a significant difference in the mean age of presentation for true bone and false bone impaction, $\mathrm{P}=0.02$. Conclusion: Due to the low sensitivity of $\mathrm{x}$-ray we do not recommend the utilisation of plain film radiography to rule out bone impaction Advances in low dose radiation multidetector CT scanners may replace plain film radiography as a screening tool.
\end{abstract}

\section{Keywords}

Fish Bone Impaction, Emergency, ENT, X-Ray, Computed Tomography

\section{Introduction}

Fish bone impaction in the upper aerodigestive tract represents a common emergency department (ED) presentation and one which frequently requires review by otolaryngology specialists. Such patients can present with variable symptomatology ranging from severe pain and odynophagia to being relatively asymptomatic. In a prospective study of fish bone impaction a "sharp pricking sensation on swallowing" had the best predictive value of any symptom (76\%) [1]. Other symptoms included pain on swallowing, foreign body sensation, pain 
on rest, blood stained saliva and a sense of obstruction [1].

The duration of symptom onset to presentation at ED also showed a correlation with the presence of fish bone on endoscopy with the yield of fish bone retrieval decreasing as the duration increased [1].

The yield of fish bone retrieval using endoscopy has been shown to decrease as the duration of presentation to ED increases [1]. In patients presenting within 12 hours of symptom onset, $60 \%$ of them were found to have fish bones, whereas patients presenting between 12 to 24 hours and 3 to 7 days yielded a bone $38 \%$ and $20 \%$ of the time respectively [1].

Several cadaveric studies have also been performed evaluating the efficacy of various imaging modalities in the detection of fish bones. A study by Lue et al. involving the plain film analysis of 10 types of fish bone in a human cadaver suggested that the visualisation of fish bones was more dependent on their position rather than their degree of radio-opacity [2].

Typically, patients presenting with suspected fish bone impaction will receive plain film radiography with an otolaryngology consult for flexible nasoendoscopy to visualise the foreign body. Utilisation of plain film radiography is controversial due to its low sensitivity [1] [2] [3]. Studies have suggested that plain film radiography has a relatively low sensitivity $(24 \%-54.8 \%)$ and high specificity (72\% - 100\%) when it comes to the detection of fish bones [4] [5] [6] [7] [8]. The low sensitivity has been shown to be related to the comparatively low densities of certain fish bones and difficulties in evaluation related to the calcification of surrounding laryngeal structures [2] [3].

Despite this x-ray has been advocated for use in the initial evaluation of fish bone impaction because of the presumed perception that it is cost-effective and leads to a quicker diagnosis with minimal radiation exposure [4] [9].

In comparison, Computer Tomography (CT) has been shown to be highly accurate in identifying fish bones with sensitivity and specificity values approaching $100 \%$ [4].

A recent study by Devarajan et al. evaluating the utility of the plain-film screen for fish and chicken bone impaction considered its efficacy in terms of the cost incurred by the hospital, patients' length of stay and radiation exposure [4]. It found plain film radiography did not reduce the cost to the hospital or length of stay in false impactions compared to true impactions. The authors went on to suggest that low dose radiation multidetector CT evaluation could serve as an alternative screening tool [4] [10].

The most common sites for fish bone impaction are the palatine tonsils, tongue base, vallecula and piriform sinus [11]. Fish bones impacted in the palatine tonsils should be detectable by direct examination [11].

As there is limited data from an Australian population and conflicting opinions of the use of plain film radiography as screening tools, the primary aim of this study was to quantify the accuracy of $\mathrm{x}$-ray and to test the perception that plain film radiography can be used to rule out fish bone impaction in the upper 
aerodigestive tract where it is suspected but not detected on direct examination [4]. Our secondary aims were to evaluate demographic factors to predict true fish bone impaction.

\section{Method}

A retrospective multicentre cohort study of patients presenting to Eastern Health Emergency Departments was conducted between January 2010 to December 2015. All patients presenting to one of either Box Hill Hospital, Maroondah Hospital or the Angliss Hospital with a possible fish impaction between the study period were included in the final analysis. Patients who did not undergo a radiological investigation and subsequent gold standard investigation were excluded from the study.

In this study, the gold standard of diagnosis was defined as direct visualisation via endoscopy (flexible nasoendoscopy or laryngoscopy). The imaging modalities were interpreted by a consultant radiologist.

Electronic and paper records from the emergency departments and hospital wards were accessed to obtain data including sex, race, age, symptomology, $\mathrm{x}$-ray performed, CT performed, onset of symptomology and time to presentation to ED, representations, Ear Nose and Throat (ENT) consultation, form of endoscopy and location of true bone impaction was also recorded.

The outcome of each of the imaging modalities was determined whether it was a true positive true negative. The sensitivities, specificities, positive predictive values (PPV) and negative predictive values (NPV) were calculated. Descriptive statistics were used to summarise the characteristics of the included patients. Frequencies and percentages were used for categorical variables. Chi Square tests were used to compare categorical variables. Mann-Whitney $U$ tests (two groups) were used to compare the means of continuous variables where data were not normally distributed. Tables and graphs were generated using Microsoft Excel to assist in interpretation of results. Statistical analysis was carried out using Graphpad Software [12]. P-values of less than 0.05 (5\%) were chosen as the level of significance.

\section{Results}

A total of 73 patients were included in the final analysis from the period of 2010 and 2015 with suspected fish bone impaction. Table 1 summarises the demographic and clinical data that were obtained during this study. A total of 28 patients $(38.4 \%)$ had true bone impaction. The majority of presentations were female in gender $(75 \%)$. There was no significant difference in terms of gender or race and bone impaction rates ( $\mathrm{P}$-value $=0.6$ and 1.0, respectively) (Table 1 ). The mean age of presentations for true bone impaction was 56.5 and for false bone impaction 46.3 (Table 1$)$. The difference was significant $(\mathrm{P}$-value $=0.02)$. The mean duration of presentation post ingestion of a foreign body was 16 hours with true bone impaction, and 24 hours for false bone impaction, this difference 
Table 1. Demographic data.

\begin{tabular}{cccc}
\hline Variable & $\begin{array}{c}\text { True Bone Impaction } \\
(\mathbf{n}=\mathbf{2 8})\end{array}$ & $\begin{array}{c}\text { False Bone impaction } \\
(\mathbf{n}=\mathbf{4 5})\end{array}$ & P-value \\
\hline Sex & 7 & 15 & 0.6 \\
male & 21 & 30 & 1 \\
female & 16 & 25 & \\
Race & 12 & 20 & 0.017 \\
asian & 56.5 & 46.3 & \\
non asian & 7 & 6 & \\
Age (average) & 24 & 43 & \\
CT ordered & 11 & 16 & \\
ENT consult & 16 & 24 & \\
Operating Theatre & & & \\
Mean Duration prior & & & \\
presentation to ED (hours) & & & \\
\hline
\end{tabular}

was not significant $(\mathrm{P}$-value $=0.11)($ Table 1$)$.

Table 2 summarises sensitivity and specificity data for plain film radiography. Of the $24 \mathrm{x}$-rays that were reported as positive for bone impaction, 12 were deemed as true impaction. The sensitivity for $\mathrm{x}$-ray was $43 \%$ and specificity was $73 \%$ with a positive predictive value of $50 \%$ and negative predictive value of $67.4 \%$.

A total of 13 patients also received a CT, of these a total of 7 were reported as positive for bone impaction with 6 being deemed as true impaction. The sensitivity of CT was $85.7 \%$, specificity $83.3 \%$, positive predictive value $85.7 \%$ and negative predictive value $83.3 \%$.

Table 3 summaries the frequency of symptomatology experienced by patients. Foreign body sensation and odynophagia were the most common symptoms observed, with a frequency of $48.4 \%$ and $33.0 \%$ respectively. Table 4 summaries sensitivity and specificity data of symptomology. The sensitivity for foreign body sensation was $75 \%$ and specificity was $37.8 \%$. The positive predictive value was $42.9 \%$ and negative predictive value $70.8 \%$. Odynophagia had a sensitivity of $43.8 \%$ and a specificity of $55.6 \%$. The positive predictive value was $41.2 \%$ and negative predictive value was $58.1 \%$.

Table 5 summaries the location of true bone impaction. The majority of bone impaction occurred in the oropharynx and laryngopharynx (77.7\%). The oral cavity had an impaction rate of $11.1 \%$ and $100 \%$ removal rate. The oropharynx had an impaction rate of $37 \%$ with a $90 \%$ removal rate and $10 \%$ dislodgement. The laryngopharynx had an impaction rate of $40.1 \%$ and $45.5 \%$ removal rate with $54.5 \%$ dislodgement. The oesophagus had an impaction rate of $11.1 \%$ and a $100 \%$ removal rate. 
Table 2. Sensitivity and specificity data of X-rays for fish bones.

\begin{tabular}{cc}
\hline Variable & Fish Bones \\
\hline Sensitivity & $42.3 \%$ \\
Specificity & $73.3 \%$ \\
Positive Predictive Value & $50.0 \%$ \\
Negative Predictive Value & $67.4 \%$ \\
\hline
\end{tabular}

Table 3. Symptomology experienced by patients.

\begin{tabular}{cc}
\hline Symptomology & Frequency $(\mathbf{N}=\mathbf{7 3})$ \\
\hline Foreign body sensation & 47 \\
Odynophagia & 32 \\
Chest pain & 1 \\
Sharp prick Sensation & 9 \\
Obstruction & 1 \\
Resting throat pain & 7 \\
\hline
\end{tabular}

Table 4. Sensitivity and specificity of symptomology.

\begin{tabular}{ccc}
\hline Variable & $\begin{array}{c}\text { Foreign Body } \\
\text { Sensation }\end{array}$ & Odynophagia \\
\hline Sensitivity & $75 \%$ & $43.8 \%$ \\
Specificity & $37.8 \%$ & $55.6 \%$ \\
Positive Predictive Value & $42.9 \%$ & $41.18 \%$ \\
Negative Predictive Value & $70.8 \%$ & $58.1 \%$ \\
\hline
\end{tabular}

Table 5. Location of true bone impaction.

\begin{tabular}{cccc}
\hline Location & Total & Removed & Dislodged \\
\hline Oral cavity & 3 & 3 & 1 \\
Oropharynx & 10 & 9 & 6 \\
Laryngopharynx & 11 & 5 & \\
esophagus & 3 & 3 & \\
\hline
\end{tabular}

\section{Discussion}

\section{Statement of Principal Findings}

This study examined 73 patients who received both plain film radiography and subsequent endoscopy. The sensitivity and specificity of plain film radiography was $43 \%$ and $73.3 \%$ respectively. These results demonstrate plain film radiography is a poor screening tool [4] [5] [6] [10]. The sensitivity and specificity of CT, $85.7 \%$ and $83.3 \%$. This demonstrates the superiority of CT over plain film radiology [4] [5] [6] [10] [12] [13]. Therefore, we cannot rule out fish bone impaction with $\mathrm{x}$-ray alone. 
A total of $51 \%$ of the patients who presented to Box Hill ED were of Asian descent. This is likely due to Box Hill town centre being in close proximity to Box Hill Hospital. Box Hill town centre has a large Asian population and contains predominantly Asians restaurants, which often incorporate small fish bones [4]. We found no statistical significant difference $(\mathrm{P}=0.8)$ between Asians and Non-Asians for true and false bone impaction rates [4].

We found a significant difference $(\mathrm{P}=0.03)$ between the mean average age of true (55.0 years old) and false bone impaction (47.1 years old). Older age was more likely to be associated with true bone impaction. Devarajan et al. have previously reported no significant difference between the two variables [4]. However Kim et al. have reported similar findings to our study [14]. This difference was thought to be related to the physiological characteristics of the oesophagus and the deterioration in swallowing movement that occurs with increasing age [14] [15].

Our study demonstrated no significant difference $(P=0.11)$ between the mean duration of presentation to ED post for true and false bone impaction rates (16 hours and 24 hours respectively). Our results disagree with Devarajan et al. Ngan et al. did however report a decrease in yield of fish bone retrieval as the duration of symptoms increased [1].

The symptomology was also analysed. The majority of patients presented with foreign body sensation and odynophagia, this is consistent with previous studies looking at fish bone impaction [1] [4]. The sensitivity for foreign body sensation was $75 \%$ was specificity $37.8 \%$. Ngan et al. reported sensitivities of $94 \%$ and specificities of $6 \%$ in literature [1]. Our study suggested foreign body sensation has a lower sensitivity for detecting true fish bone impaction, however our calculated specificity was higher. Odynophagia had a sensitivity of $43.75 \%$ and specificity of $55.6 \%$. Again these results disagree with previously reported sensitivities of $83 \%$ and $21 \%$ [1].

Out of the 101 patients who presented with a complaint of bone impaction only 28 did not receive $\mathrm{x}$-rays. This demonstrates the reliance of plain film radiology as a screening tool in our emergency departments and in literature [3] [4] [9] [16].

There were four patients who represented to our emergency departments after being initially being discharged. Only one patient did not undergo $\mathrm{x}$-ray on first presentation, however this same patient underwent $\mathrm{x}$-ray on second presentation. She had successful fish bone retrieval despite having an $\mathrm{x}$-ray negative for fish bone impaction. None of the four patients underwent CT scanning.

The strengths of this study include that it was a multicentre cohort study. The study design, inclusion criteria, sample number, variables and outcomes measured are all consistent with previous studies. This allows the results of our study to be comparable to current literature.

There are several limitations of this study. Firstly, this is a retrospective study. Secondly the species of fish was not always documented. There is evidence to 
suggest the radiopacity of fish bones can depend on the species [17] [18] [19]. However, there is also evidence to suggest plain film radiography has poor sensitivity irrespective to inter-species variation in bone radiopacity [2] [3].

\section{Conclusions}

Although x-ray has a low radiation dose (0.2 mSv) [20] and cost in comparison to CT scanning [4], due to the low sensitivity of $\mathrm{x}$-ray we do not recommend the utilisation of plain film radiography to rule out bone impaction in the upper aerodigestive tract.

The results from our pilot study have demonstrated discrepancies in literature, with regards to symptomology, mean duration prior to presentation to $\mathrm{ED}$ (hours) and average age and true bone impaction. Due to these results and the limited Australian data available, we propose a multicentre prospective study to further evaluate the efficacy of plain film $\mathrm{x}$-ray in ruling out bone impaction in an Australian population. Further studies utilizing low dose CT evaluation also need to be conducted. Until then we recommend an ENT consult with flexible nasoendos copy which has previously proven to be a safe effective and economic algorithm in the management of patients with suspected bone impaction [1].

\section{Declaration of Conflicting Interests}

The author(s) declared no potential conflicts of interest with respect to the authorship, research and publication of this article.

\section{Funding}

The author(s) received no financial support for the research, authorship, and/or publication of this article.

\section{Ethics}

Ethics approval was obtained from the Office of Research and Ethics, Eastern Health. Reference number: LR08/2016.

\section{Supplementary Material}

The data was obtained through Symphony software, and electronic medical records through Eastern Health, Box Hill, Victoria.

\section{References}

[1] Ngan, J.H., et al. (1990) A Prospective Study on Fish Bone Ingestion. Experience of 358 Patients. Annals of Surgery, 211, 459-462. https://doi.org/10.1097/00000658-199004000-00012

[2] Lue, A.J., Fang, W.D. and Manolidis, S. (2000) Use of Plain Radiography and Computed Tomography to Identify Fish Bone Foreign Bodies. Otolaryngology-Head and Neck Surgery, 123, 435-438. https://doi.org/10.1067/mhn.2000.99663

[3] Ritchie, T. and Harvey, M. (2010) The Utility of Plain Radiography in Assessment 
of Upper Aerodigestive Tract Fishbone Impaction: An Evaluation of 22 New Zealand Fish Species. The New Zealand Medical Journal (Online), 123.

[4] Devarajan, K., et al. (2015) Diagnosing Fish Bone and Chicken Bone Impactions in the Emergency Department Setting: Measuring the System Utility of the Plain Film Screen. Annals of Otology, Rhinology \& Laryngology, 124, 614-621. https://doi.org/10.1177/0003489415573072

[5] Palme, C.E., Lowinger, D. and Petersen, A.J. (1999) Fish Bones at the Cricopharyngeus: A Comparison of Plain-Film Radiology and Computed Tomography. Laryngoscope, 109, 1955-1958. https://doi.org/10.1097/00005537-199912000-00011

[6] Watanabe, K., et al. (1998) The Usefulness of Computed Tomography in the Diagnosis of Impacted Fish Bones in the Oesophagus. The Journal of Laryngology \& Otology, 112, 360-364. https://doi.org/10.1017/S0022215100140460

[7] Qureshi, T.A., et al. (2017) Effectiveness of Plain X-Ray in Detection of Fish and Chicken Bone Foreign Body in Upper Aerodigestive Tract. JPMA: Journal of The Pakistan Medical Association, 67, 544.

[8] Kim, H.U. (2016) Oroesophageal Fish Bone Foreign Body. Clinical Endoscopy, 49, 318. https://doi.org/10.5946/ce.2016.087

[9] Carr, A. (1987) Radiology of Fish Bone Foreign Bodies in the Neck. The Journal of Laryngology \& Otology, 101, 407-408. https://doi.org/10.1017/S0022215100101896

[10] Park, S., et al. (2014) Fish Bone Foreign Bodies in the Pharynx and Upper Esophagus: Evaluation with 64-Slice MDCT. Acta Radiologica, 55, 8-13. https://doi.org/10.1177/0284185113493087

[11] Hone, S.W., et al. (1995) The Radio-Opacity of fishbones: A Cadaveric Study. Clinical Otolaryngology and Allied Sciences, 20, 234-235. https://doi.org/10.1111/j.1365-2273.1995.tb01855.x

[12] Inc, G.S. (2016) QuickCalcs. http://www.graphpad.com/quickcalcs/

[13] Woo, S.H. and Kim, K.H. (2015) Proposal for Methods of Diagnosis of Fish Bone Foreign Body in the Esophagus. The Laryngoscope, 125, 2472-2475. https://doi.org/10.1002/lary.25340

[14] Kim, J.P., et al. (2015) Analysis of Clinical Feature and Management of Fish Bone Ingestion of Upper Gastrointestinal Tract. Clinical and Experimental Otorhinolaryngology, 8, 261-267. https://doi.org/10.3342/ceo.2015.8.3.261

[15] Sheth, N. and Diner, W.C. (1988) Swallowing Problems in the Elderly. Dysphagia, 2, 209-215. https://doi.org/10.1007/BF02414428

[16] Evans, R., et al. (1992) The Lateral Neck Radiograph in Suspected Impacted Fish Bones-Does It Have a Role? Clinical Radiology, 46, 121-123. https://doi.org/10.1016/S0009-9260(05)80316-2

[17] Davies, W.R. and Bate, P.J. (2009) Relative Radio-Opacity of Commonly Consumed Fish Species in South East Queensland on Lateral Neck X-Ray: An Ovine Model. Medical Journal of Australia, 35, 677-680.

[18] Kumar, M., et al. (2003) Fish Bone as a Foreign Body. The Journal of Laryngology \& Otology, 117, 568-569. https://doi.org/10.1258/002221503322113058

[19] Koornhof, P., Du Plessis, L. and Claassen, A. (1990) Swallowed Fish Bones-Is Radiography Justified? A Case Report. South African Journal of Surgery, 28, 158-160.

[20] Mettler Jr., F.A., et al. (2008) Effective Doses in Radiology and Diagnostic Nuclear Medicine: A Catalog 1. Radiology, 248, 254-263. https://doi.org/10.1148/radiol.2481071451 